\title{
INTENSIDADE ÓTIMA DE PODA EM Eucalyptus dunnii PARA A PRODUÇÃO DE MADEIRA LIVRE DE NÓS
}

\author{
Mário Dobner Junior ${ }^{1 *}$, Rudi Arno Seitz ${ }^{2}$ \\ ${ }^{1 *}$ Universidade Federal de Santa Catarina, Curitibanos, Santa Catarina, Brasil - mario.dobner@ufsc.br \\ ${ }^{2}$ Universidade Federal do Paraná, Curitiba, Panará, Brasil, in memoriam
}

Recebido para publicação: 14/01/2015 - Aceito para publicação: 12/08/2015

\begin{abstract}
Resumo
Eucalytpus dunnii é uma espécie subtropical e tem seu cultivo indicado para regiões onde há ocorrência de geadas. Apesar de ser atualmente utilizada para a produção de papel, carvão e chapas, o uso sólido da madeira para fins mais nobres é conhecido, desde que práticas silviculturais como podas e desbastes sejam consideradas. O presente estudo teve como objetivo analisar o crescimento de indivíduos dominantes de E. dunnii submetidos a diferentes intensidades de poda. Foram testadas quatro diferentes intensidades de poda, mantendo uma copa remanescente de 100, 80, 50 e $20 \%$ da altura total de árvores dominantes. Foi utilizado um delineamento inteiramente casualizado com 10 indivíduos por tratamento. Passados 20 meses da realização da poda, a manutenção de até $50 \%$ da copa foi suficiente para manter um crescimento em diâmetro considerado normal. Mais importante que a proporção de copa remanescente, é o comprimento absoluto das mesmas. Conclui-se que são necessários, no mínimo, $4 \mathrm{~m}$ de copa remanescente para que as árvores mantenham um incremento periódico anual acima de $4 \mathrm{~cm}$ (média anual durante o período de 17 a 37 meses). Ao ultrapassar este limite, perdas na ordem de $50 \%$ do incremento em diâmetro são esperadas.
\end{abstract}

Palavras-chave: Desrama; Manejo Florestal; Múltiplos usos.

\begin{abstract}
Optimum pruning intensity in Eucalytpus dunnii for clear wood producing. Eucalyptus dunnii is a subtropical species, indicated for areas where frosts occur. Besides its current utilization for paper, coal and boards production, the potential for nobler solid-uses are recognized, provided that silvicultural practices such as pruning and thinning are regarded. The objective of this study was to analyze the growth of dominant $E$. dunnii trees subjected to four different pruning intensities: 100, 80, 50 and $20 \%$ of the total tree height of remained canopy. Experimental design was fully randomized, with 10 replications per treatment. After 20 months of pruning, maintaining 50\% of canopy was sufficient to allow a normal diameter growth. More important than the proportion, was the absolute length of remained canopy. It was concluded that it is necessary to keep, at least, $4 \mathrm{~m}$ of remained canopy in order to maintain a periodic annual increment over $4 \mathrm{~cm}$ (average during the 17-37 months period). When this threshold is exceeded, lost in the order of $50 \%$ in diameter increment are expected.
\end{abstract}

Keywords: Forest Management; Multiple-uses.

\section{INTRODUÇÃO}

Eucalyptus dunnii Maiden ocorre naturalmente na costa sudeste da Austrália, onde o clima é subtropical úmido. No planalto Sul do Brasil vem sendo amplamente cultivado em altitudes variando entre 500 e $1.000 \mathrm{~m}$ acima do nível do mar (a.n.m.), por apresentar bom crescimento e moderada tolerância às geadas (HIGA et al., 2000, PALUDZYSZYN; SANTOS, 2005; DOBNER JR. et al. 2009).

A madeira é atualmente utilizada no abastecimento de indústrias de celulose e na produção de carvão vegetal e chapas. Entretanto, o potencial para usos mais nobres de maior valor agregado é conhecido (PEREYRA, 1994; HARWOOD et al., 2005; ROCHA; TRUGILHO, 2006; GLENCROSS et al., 2011), embora algumas limitações tenham sido relatadas (JOVANOVIC et al., 2000; TRUGILHO; SILVA, 2008). No Brasil usos mais nobres da madeira de Eucalyptus ainda são restritos, apesar de ser amplamente utilizado no mercado australiano de madeira sólida, particularmente para pisos, acabamentos internos e móveis (WOUGH, 2004). 
Estudos anteriores afirmam que a produção de madeira sólida de espécies de Eucalyptus passa, necessariamente, por programas específicos de melhoramento genético (SHIELD, 2004; HARWOOD et al., 2005; LIMA et al., 2005; NOLAN et al., 2005; HAGUE, 2013). Juntamente com o melhoramento genético, práticas silviculturais apresentam potencial para melhorar a qualidade da madeira. Desbastes e poda são atividades imprescindíveis neste contexto (MONTAGNU et al., 2003; SHIELD, 2004; NUTTO; TOUZA VÁZQUEZ, 2004; NUTTO et al., 2006; WASHUSEN, 2013).

A realização da poda é frequentemente questionada no que diz respeito aos reais benefícios econômicos. Por isso, quando há a intenção de fazê-la, é necessário otimizar a operação de forma a aumentar a sua eficiência desta. Uma recomendação comum é podar o maior segmento possível em uma única intervenção de forma a otimizar o deslocamento das equipes de trabalhadores. Sabe-se, porém, que uma remoção drástica dos galhos implica em perda da capacidade fotossintética das árvores e, consequentemente, redução do crescimento.

Há, portanto, necessidade de se definir critérios para orientar decisões neste sentido. O presente estudo teve como objetivo analisar o crescimento de indivíduos dominantes de E. dunnii submetidos a diferentes intensidades de poda.

\section{MATERIAL E MÉTODOS}

O presente estudo foi desenvolvido em povoamento de $E$. dunnii estabelecido com uma densidade inicial de 1.600 árvores por hectare (2,5 x 2,5 m), no município de Campo Belo do Sul, SC. A área de estudo estava localizada a $940 \mathrm{~m}$ a.n.m. (Lat. $27^{\circ} 59^{\prime} 44^{\prime \prime} \mathrm{S}$, Long. $50^{\circ} 51^{\prime} 51^{\prime \prime}$ O). A precipitação média anual é de $1.700 \mathrm{~mm}$, com temperatura média anual de $16^{\circ} \mathrm{C}$. A temperatura média do mês mais frio varia entre $5-8^{\circ} \mathrm{C}$, com a ocorrência de 2 a 29 geadas por ano (EMBRAPA, 1988).

Antes da implantação do povoamento de $E$. dunnii, o solo (Cambissolo) era utilizado para a produção de culturas anuais. O plantio do povoamento foi realizado no mês de setembro de 2003.

Após o plantio, procedeu-se adubação com $240 \mathrm{~kg} \mathrm{ha}^{-1}$ de NPK, na formulação 5:20:10, incorporanda ao solo ao redor das mudas.

Aos 17 meses de idade, no mês de março, o experimento foi iniciado. Indivíduos dominantes de E. dunnii foram selecionados e submetidos a 4 diferentes intensidades de poda, com a remoção de galhos até $0,20,50$ e $80 \%$ da altura total das árvores, resultando comprimentos relativos de copa remanescente de $100,80,50$ e $20 \%$, respectivamente. O tratamento com $100 \%$ de copa remanescente foi considerado a testemunha, por manter toda a copa das árvores.

O tratamento controle, onde $100 \%$ da copa foi mantida, não significa que a árvore possuía $100 \%$ da altura total de copa verde. Em função da competição entre indivíduos e envelhecimento do povoamento, no momento da realização da poda estas árvores possuíam, na média, 90\% da altura total de copa verde.

Ao todo, 10 indivíduos por tratamento foram avaliados. O delineamento experimental utilizado foi o inteiramente casualizado. Informações detalhadas sobre os indivíduos em cada tratamento são apresentados na tabela 1 .

Tabela 1. Características das árvores aos 17 meses de idade, nas diferentes intensidades de poda analisadas. Altura da base da copa verde após a realização da poda. Intervalos de confiança considerando $95 \%$ da distribuição.

Table 1. Tree characteristics at 17 months age, for the different pruning intensities. Live-canopy height after pruning. Confidence intervals regarding $95 \%$ of the distribution.

\begin{tabular}{lcccc}
\hline $\begin{array}{l}\text { Comprimento de copa } \\
\text { remanescente (\%) }\end{array}$ & $\begin{array}{c}\mathbf{N}^{\circ} \\
\text { árvores }\end{array}$ & $\begin{array}{c}\text { DAP } \\
(\mathbf{c m})\end{array}$ & $\begin{array}{c}\text { Altura } \\
(\mathbf{m})\end{array}$ & $\begin{array}{c}\text { Altura da base da copa } \\
\text { verde após a poda (m) }\end{array}$ \\
\hline 100 & 10 & $8,1 \pm 1,0$ & $7,1 \pm 1,4$ & $0,6 \pm 0,8$ \\
80 & 10 & $7,7 \pm 0,7$ & $6,8 \pm 1,0$ & $1,5 \pm 0,2$ \\
50 & 10 & $8,1 \pm 2,2$ & $7,0 \pm 1,3$ & $3,6 \pm 0,8$ \\
20 & 10 & $8,2 \pm 1,9$ & $7,0 \pm 1,0$ & $5,0 \pm 0,3$ \\
\hline $95 \% \cdot 1,96 *$ desvio padrão $(S)$ & & & &
\end{tabular}

As alturas de poda foram definidas individualmente, de acordo com a altura total de cada árvore, mantendo a proporcionalidade na intensidade de remoção da copa. 
A poda foi realizada com serrote manual, adaptado com cabo de madeira quando a altura excedia o alcance do trabalhador.

Foram avaliados o crescimento em Diâmetro a Altura do Peito (DAP) e em altura total aos 17, quando da realização dos tratamentos de poda, e aos 37 meses de idade do povoamento, 20 meses após as árvores terem recebido os tratamentos com as diferentes intensidades de poda.

\section{RESULTADOS}

Os valores de DAP e de altura total obtidos aos 17 e 37 meses de idade, respectivamente, para as diferentes intensidades de poda, são apresentados na tabela 2 .

Tabela 2. Diâmetro a altura do peito (DAP) e altura total das árvores submetidas às diferentes intensidades de poda, obtidos aos 17 e aos 37 meses de idade.

Table 2. Trees diameter at breast height $(\mathrm{DBH})$ and total height as affected by the different pruning intensities, measured at age 17 and 37 months.

\begin{tabular}{lcccc}
\hline $\begin{array}{l}\text { Comprimento de copa } \\
\text { remanescente (\%) }\end{array}$ & $\begin{array}{c}\text { DAP 17 meses } \\
(\mathbf{c m})\end{array}$ & $\begin{array}{c}\text { DAP 37 meses } \\
(\mathbf{c m})\end{array}$ & $\begin{array}{c}\text { Altura 17 meses } \\
(\mathbf{m})\end{array}$ & $\begin{array}{c}\text { Altura 37 meses } \\
(\mathbf{m})\end{array}$ \\
\hline 100 & $8,1 \mathrm{a}$ & $17,0 \mathrm{a}$ & $7,2 \mathrm{a}$ & $14,9 \mathrm{a}$ \\
80 & $7,7 \mathrm{a}$ & $17,0 \mathrm{a}$ & $6,8 \mathrm{a}$ & $14,6 \mathrm{ab}$ \\
50 & $8,1 \mathrm{a}$ & $15,4 \mathrm{a}$ & $7,0 \mathrm{a}$ & $14,5 \mathrm{ab}$ \\
20 & $8,2 \mathrm{a}$ & $12,8 \mathrm{~b}$ & $7,0 \mathrm{a}$ & $13,2 \mathrm{~b}$ \\
\hline$P$ & 0,59 & $<0,01$ & 0,71 & 0,04 \\
\hline$p$ probabilidade. As médias seguidas pela mesma letra no sentido vertical não diferem entre si.
\end{tabular}

Analisando-se a tabela 2, constata-se que aos 17 meses, tanto o DAP como a altura das árvores selecionadas para os diferentes tratamentos de poda não apresentaram diferenças significativas entre os grupos. Esta observação permite inferir que todas as diferenças futuras são decorrentes das intensidades de remoção de copa promovida pelos tratamentos de poda testados.

Com relação ao DAP, verifica-se que, passados 20 meses da realização da poda, a manutenção de até 50\% da copa foi suficiente para manter um crescimento considerado normal, semelhante ao obtido em árvores não podadas (100\% de copa remanescente).

Para um melhor entendimento dos efeitos da poda no crescimento em diâmetro das árvores, apresenta-se na figura 1, o incremento periódico anual (IPA) dos 20 meses posteriores à intervenção em relação à proporção (\%) e ao comprimento $(\mathrm{m})$ de copa remanescente.
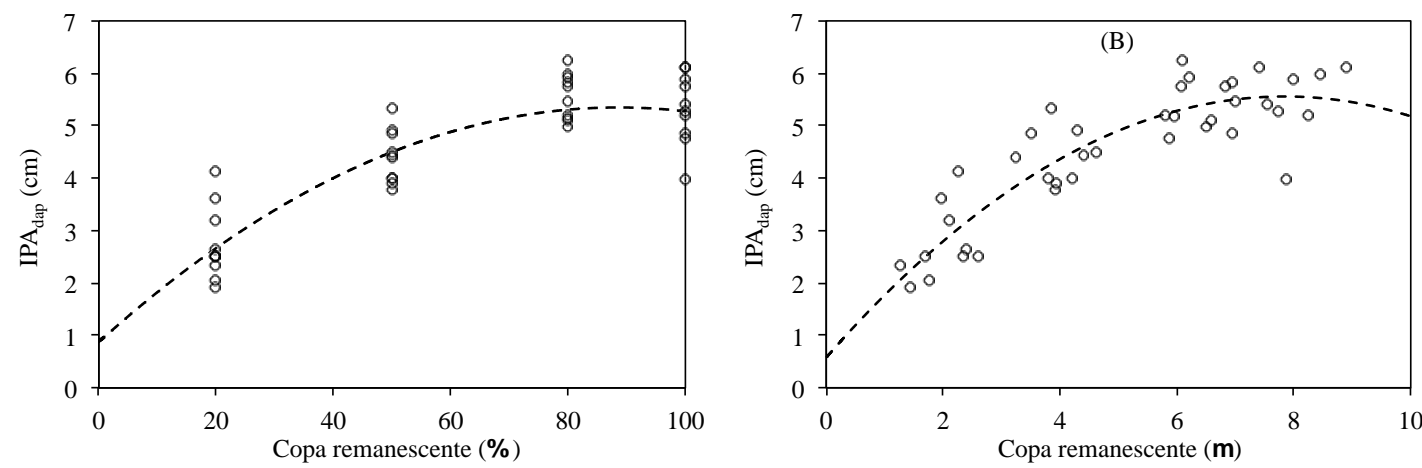

Figura 1. Incremento periódico anual (IPA) em cm durante o período de 17 a 37 meses em relação (A) à proporção (\%) e (B) ao comprimento (m) de copa remanescente. Os modelos de regressão para ambas as situações são apresentados abaixo:

Figure 1. Periodic annual increment (PAI) in $\mathrm{cm}$ during the 17-37-months period in relation to (A) the proportion (\%) and (B) to the length (m) of the remained canopy. Regression models for both situations are given below:

FLORESTA, Curitiba, PR, v. 45, n. 4, p. 791 - 796, out. / dez. 2015

Dobner Junior, M.; Seitz, R. A.

ISSN eletrônico 1982-4688 / ISSN impresso 0015-3826

DOI: $10.5380 /$ rf.v45i4.39474 


$$
\begin{array}{lll}
I P A_{\text {dap }}=0,875+0,101\left(C R_{\%}\right)-0,00057\left(C R_{\%}\right)^{2} & \mathrm{R}^{2}=0,78 & \mathrm{~S}_{\mathrm{yx}}=13,0 \% \\
I P A_{\text {dap }}=0,580+1,270\left(C R_{m}\right)-0,081\left(C R_{m}\right)^{2} & \mathrm{R}^{2}=0,85 & \mathrm{~S}_{\mathrm{yx}}=11,0 \%
\end{array}
$$

Reforça-se a necessidade de manter, pelo menos, 50\% da copa verde das árvores para permitir incrementos em diâmetros nos mesmos níveis de árvores não podadas (Figura 1-A).

Mais importante que a proporção de copa remanescente, é o comprimento absoluto das mesmas (Figura 1-B). Verifica-se que são necessários, no mínimo, $4 \mathrm{~m}$ de copa remanescente para que as árvores mantenham um incremento periódico anual acima de $4 \mathrm{~cm}$ (média anual durante o período de 17 a 37 meses). Ao ultrapassar este limite, perdas na ordem de 50\% do incremento em diâmetro são esperadas.

Com relação ao crescimento em altura, constatou-se diferenças significativas somente para as árvores com menos de 50\% de copa remanescente $(p<0,01)$. Enquanto árvores com mais de $50 \%$ de copa remanescente cresceram $\sim 4,6 \mathrm{~m}$ em altura por ano após a realização da poda, árvores com $20 \%$ de copa remanescente apresentaram um incremento periódico anual em altura total de apenas $3,7 \mathrm{~m}$.

\section{DISCUSSÃO}

A poda de árvores para a produção de madeira livre de nós é uma atividade bastante estudada para povoamentos de Pinus. Para Eucalyptus, raros são os estudos publicados, principalmente com espécies subtropicais, como é o caso de $E$. dunnii, recomendadas para o cultivo em regiões com a ocorrência de geadas.

Dos poucos trabalhos existentes, a discussão sobre a quantidade de copa viva remanescente é preponderantemente tratada de forma relativa. Recomenda-se manter não menos de 40-50\% de copa remanescente da altura total dos indivíduos (COUTO, 1995; SMITH; BRENNAN, 2006; NUTTO et al., 2006). Os resultados apresentados na figura 1 evidenciam o problema relacionado com uma abordagem unicamente relativa. A prática de orientar os trabalhadores rurais para manter uma copa remanescente igual a 50\% da altura total da árvore, independente do comprimento absoluto da mesma, mostra-se equivocada. Ao utilizar este procedimento para podar uma árvore com $5 \mathrm{~m}$ de altura, por exemplo, restariam apenas $2,5 \mathrm{~m}$ de copa. De acordo com o observado na figura 1-B, insuficiente para manter um crescimento em diâmetro considerado normal.

Para que as árvores podadas continuem crescendo de forma semelhante às não podadas, ou seja, para que elas expressem todo seu potencial produtivo, é necessário manter mais de $4 \mathrm{~m}$ de copa.

Aos 17 meses de idade, quando a poda foi realizada, as árvores apresentavam uma altura média de $7 \mathrm{~m}$. A base da copa verde, ou o segmento da copa que secou em função da competição entre indivíduos era de 0,6 m (0,2-1,4 m). Considerando o limite mínimo de $4 \mathrm{~m}$ remanescentes, conclui-se que a altura da poda não deveria exceder os $3 \mathrm{~m}$. Desta forma, mantendo um ritmo de crescimento considerado normal.

Sabendo-se da necessidade de manutenção de copa remanescente, e do crescimento das árvores dominantes nos diferentes sítios, é possível prescrever a poda de forma consciente a equilibrar o ótimo silvicultura e operacional. Por exemplo, em sítios mais ou menos produtivos que o objeto de estudo, em função das condições edáficas, adubação, material genético, etc., o momento da realização da poda precisa ser analisado pontualmente. Adicionalmente, deve-se ter em mente que a poda pode não ser interessante em sítios pouco produtivos.

Os resultados obtidos no presente estudo diferem dos relatados por Finger et al. (2001) para $E$. saligna plantado na região Leste do Rio Grande do Sul, com altitude de $85 \mathrm{~m}$ a.n.m. Os autores não detectaram nenhuma redução do crescimento em árvores podadas até $80 \%$ da altura total (1,5 m de copa remanescente). Apesar das características dendrométricas das árvores no momento da realização da poda serem semelhantes entre os dois estudos, as condições climáticas mais favoráveis no trabalho citado podem ter contribuído para uma melhor recuperação das árvores.

Podas excessivas são particularmente problemáticas, pois esta atividade é empregada quando o objetivo é justamente concentrar a produção em um número reduzido de indivíduos selecionados. Sabe-se que a produção de madeira livre de nós é consequência do diâmetro do núcleo nodoso (diâmetro máximo do fuste no momento da poda, acrescido a faixa de oclusão) e do incremento em diâmetro após a poda. 
Ao influenciar negativamente o incremento em diâmetro com a poda, há, obviamente, um conflito de objetivos e prejuízos econômicos.

O presente estudo avaliou a influência da poda apenas nos indivíduos dominantes, pois acreditase que esta seja uma atividade que deva ser realizada somente nas melhores árvores, que permanecerão até o corte raso. Desta forma, o investimento adicional com a poda seria otimizado.

Segundo Nutto et al. (2006), para a produção de madeira de Eucalyptus de alta qualidade, devese podar os galhos ainda vivos, o que reduz o tempo de cicatrização e a formação de bolsas de goma.

Com a necessidade de se podar precocemente as árvores, de forma a garantir a remoção dos galhos ainda vivos, e considerando o exposto na tabela 1, onde a base da copa verde das árvores não podadas variavam de 0,2 a 1,4 m de altura, constata-se que a poda realizada aos 17 meses de idade pode ser considerada atrasada. Muito embora pareça ser apropriada considerando o desenvolvimento em altura e a possibilidade de se podar, na média, até $3 \mathrm{~m}$ em uma única intervenção. Uma alternativa neste contexto seria ampliar o espaçamento de plantio para aumentar a luminosidade e, assim, permitir que as árvores mantenham os galhos basais vivos por mais tempo.

Aos 37 meses de idade das árvores, quando elas possuíam, em média, uma altura total de $15 \mathrm{~m}$, observou-se que a base da copa verde era de 2,1 m (0,5-4,4 m). Mesmo nos indivíduos com maior perda de copa verde, os galhos permaneciam fixados ao fuste, em processo de incorporação. Estas observações indicam que a desrama natural, apesar de relatada para Eucalyptus, em E. dunnii, é insuficiente e ineficiente para garantir a produção de madeira livre de nós, reforçando a necessidade da poda artificial.

Embora não apresentadas, medições intermediárias foram realizadas, aos 21 e 32 meses de idade do povoamento. A análise dos incrementos periódicos aos 21 meses (4 meses após a realização da poda) indicou que os efeitos negativos da remoção excessiva da copa foram imediatos, principalmente com relação ao incremento em diâmetro. Enquanto as árvores com 50\% ou mais de copa $(\sim 4 \mathrm{~m})$ cresceram $1,6 \mathrm{~cm}$ em diâmetro, as árvores com apenas $20 \%(\sim 2,5 \mathrm{~m})$ de copa remanescente cresceram apenas $0,5 \mathrm{~cm}$, menos de um terço do crescimento considerado normal.

Pelo menos no médio prazo, descarta-se a hipótese de que as árvores poderiam se recuperar e retomar um ritmo de crescimento considerado normal. De qualquer forma, mesmo que as árvores se recuperem em algum momento, o crescimento perdido não será compensado.

Apesar da classificação sociológica das árvores ser mais difícil quanto mais jovem os indivíduos, constatou-se que a seleção das árvores dominantes para a poda aos 17 meses de idade foi eficiente, pois estas mantiveram a posição de dominância durante todo o período de estudo. Mesmo as árvores com apenas $20 \%$ de copa remanescente, com perdas significativas de crescimento em diâmetro e altura, não foram dominadas pelas árvores vizinhas, muito embora tenham perdido a superioridade anteriormente evidente.

\section{CONCLUSÕES}

- Conclui-se que a poda de indivíduos dominantes de E. dunnii deve ser planejada de forma a manter não menos de 4 metros de copa remanescente. Caso este limite seja ultrapassado, perdas consideráveis (50\%) no crescimento em diâmetros dos indivíduos são esperadas.

\section{REFERÊNCIAS}

DOBNER JR., M.; HIGA, A. R.; SEITZ, R. A. Efeito da cobertura de Pinus taeda na proteção contra geadas e no crescimento de plantas jovens de Eucalyptus dunnii Maiden. Revista Floresta, v. 39, n. 4, p. $807-823,2009$.

EMPRESA BRASILEIRA DE PESQUISA AGROPECUÁRIA (EMBRAPA). Centro Nacional de Pesquisas Florestais (CNPF). Zoneamento ecológico para plantios florestais no estado de Santa Catarina. Curitiba, 1988. 113 p.

FINGER, C. A. G.; SCHNEIDER, P. R.; BAZZO, J. L.; KLEIN, J. E. M. Efeito da intensidade de desrama sobre o crescimento e a produção de Eucalytpus saligna Smith. Revista Cerne, v. 7, n. 2, p. 53 64, 2001. 
GLENCROSS, K.; PALMER, G.; PELLETIER, M. C.; NICHOLS, J. D. Growth response to thinning in two subtropical hardwood species. Technical Report 217. Cooperative Research Centre for Forestry, Hobart, 2011. 27 p.

HAGUE, J. R. B. Utilization of plantation eucalyptus in engineered wood products. Forest \& Wood products Australia, Project no. PNB290-1112, Melbourne, 2013. 20 p.

HARWOOD, C.; BANDARA, K.; WASHUSEN, R.; NORTHWAY, R.; HENSON, M.; BOYTON, S. Variation in wood properties of plantation-grown Eucalyptus dunnii relevant to solid-wood products. Forest and Wood Products Research and Development Corporation, Project no. PN04.3003, Victoria, 2005, $40 \mathrm{p}$.

HIGA, R. C. V.; HIGA, A. R.; TREVISAN, R.; SOUZA, M. V. R. Resistência e resiliência a geadas em Eucalyptus dunnii Maiden plantados em Campo do Tenente, PR. Boletim de Pesquisas Florestais, Colombo, n. 40, p. 67 - 76, 2000.

MONTAGU, K. D.; KEAMEY, D. E.; SMITH, R. G. B. The biology and silviculture of pruning planted eucalypts for clear wood production - a review. Forest Ecology and Management, n. 179, p. 1 - 13, 2003.

NOLAN, G.; GREAVES, B.; WASHUSEN, R.; PARSONS, M.; JENNINGS, S. Eucalypt plantations for solid wood products in Australia - A review. Project No. PN04.3002. Forest and Wood Products Research and Development Corporation, Melbourne, 2005. 138 p.

NUTTO, L.; SPATHELF, P.; SELING, I. Management of individual tree diameter growth and implications for pruning for Brazilian Eucalyptus grandis HILL ex MAIDEN. Revista Floresta, v. 36, n. 3, p. 397 - 413, 2006.

NUTTO, L.; TOUZA VÁZQUEZ, M. C. High Quality Eucalypt Sawlog Production. In: IUFRO CONFERENCE "EUCALYPTUS IN A CHANGING WORLD”, 2004, Aveiro, Portugal. Proceedings... Aveiro, 2004. p. 658 - 666.

PALUDZYSZYN, E. F.; SANTOS, P. E. T. Considerações sobre o plantio de Eucalyptus dunnii no estado do Paraná. Colombo. Embrapa Florestas. Comunicado Técnico 141, 2005.

PEREYRA, O. Avaliação da madeira de Eucalyptus dunnii na manufatura de painéis compensados. 102 f. Dissertação (Mestrado em Ciências Florestais) - Escola Superior de Agricultura "Luiz de Queiroz", Universidade de São Paulo, Piracicaba, 1994.

ROCHA, M. P.; TRUGILHO, P. F. Qualidade de madeira serrada de Eucalyptus dunnii em função do método de desdobro e condição de umidade. Revista Cerne, Lavras, v. 12, n. 4, p. 314 - 321, 2006.

SHIELD, E. Silviculture for sawlog - a review of the key elements, with special reference to Eucalyptus grandis. In: IUFRO CONFERENCE "EUCALYPTUS IN A CHANGING WORLD", 2004, Aveiro, Portugal. Proceedings... Aveiro, 2004. p. 62 - 67.

SMITH, R. G. B.; BRENNAN, P. First thinning in subtropical eucalypt plantation grown for high-value solid wood products: a review. Australian Forestry, v. 69, n. 4, p. 305 - 312. 2006.

WASHUSEN, R. Processing methods for production of solid wood products from plantation-grown eucalyptus species of importance to Australia. Forest \& Wood Products Australia, Project no. PNB2911112A, Melbourne, 2013. 58 p.

WOUGH, G. Growing Eucalyptus globulus for high quality sawn products. In: IUFRO CONFERENCE "EUCALYPTUS IN A CHANGING WORLD", 2004, Aveiro, Portugal. Proceedings... Aveiro, 2004. p. $79-84$. 